\title{
Experimental oscillator strengths in Th II
}

\author{
H. Nilsson ${ }^{1}$, Z. G. Zhang ${ }^{2}$, H. Lundberg ${ }^{2}$, S. Johansson ${ }^{1}$, and B. Nordström ${ }^{1,3}$ \\ 1 Atomic Astrophysics, Lund Observatory, Lund University, PO Box 43, 22100 Lund, Sweden \\ 2 Atomic Physics, Department of Physics, Lund Institute of Technology, PO Box 118, 22100 Lund, Sweden \\ 3 Niels Bohr Institute for Astronomy, Physics \& Geophysics, Juliane Marie vej 30, 2100 Copenhagen, Denmark \\ e-mail: zhang.zhiguo@fysik.lth.se; hans.lundberg@fysik.lth.se; sveneric.johansson@astro.lu.se; \\ birgitta@astro.ku.dk
}

Received 17 October 2001 / Accepted 5 November 2001

\begin{abstract}
We have measured radiative lifetimes of ten Th II levels by using the laser-induced fluorescence technique and branching fractions with Fourier transform spectroscopy. By combining the new branching fractions with a total of 23 lifetimes, from the present work and from measurements by Simonsen et al. (1988), absolute oscillator strengths for 180 lines have been derived. Some of these new $f$-values reported are relevant for radioactive dating of stars.
\end{abstract}

Key words. atomic data - stars: evolution - Galaxy: evolution

\section{Introduction}

One way of estimating the age of the Galaxy is radioactive dating of stars. The idea of such a cosmochronometer is to compare the change with time of the abundance ratio of a radioactive element and a stable element. The present ratio derived from the star's spectrum is compared with the abundance ratio at the formation of the star, predicted from a model. In a recent paper, Cayrel et al. (2001) report the age of a metal deficient star as determined with a uranium-thorium cosmochronometer. The radioactive thorium and uranium isotopes present in the star have half-lifes of $14 \mathrm{Gyr}$ and $4.5 \mathrm{Gyr}$, respectively. A major part of the uncertainty of the age derived for the star is associated with the oscillator strengths of Th II and U II lines, used in the abundance determinations. This paper deals with $f$-value data for Th II, which has the ground configuration $6 \mathrm{~d}^{2}\left({ }^{3} \mathrm{~F}\right) 7 \mathrm{~s}$. The lowest excited configurations are $5 \mathrm{f}\left({ }^{2} \mathrm{~F}\right) 7 \mathrm{~s}^{2}$ and $5 \mathrm{f} 6 \mathrm{~d}\left({ }^{3} \mathrm{H}\right) 7 \mathrm{~s}$.

Oscillator strengths in Th II have been reported by Corliss \& Bozman (1962), who measured relative line intensities in an arc spectrum. Corliss (1979) renormalized the intensities using the radiative lifetime of one level measured by Andersen \& Petkov (1975). Later Palmer \& Engelman (1983) measured relative intensities and accurate wavelengths from a hollow cathode $(\mathrm{HC})$ discharge and normalized the intensities to the $g f$-values of Corliss \& Bozman (1962) by assuming local thermodynamic equilibrium (LTE) in the light source. Simonsen et al. (1988)

\footnotetext{
Send offprint requests to: $\mathrm{H}$. Nilsson,
}

e-mail: hampus.nilsson@astro.lu.se measured lifetimes for 18 levels in Th II and compared with lifetimes that can be derived from the data presented by Palmer \& Engelman (1983). This comparison led Simonsen et al. (1988) to give a formula, which adjusts the $g f$-values of Corliss (1979). This adjustment results, however, in an inconsistency: The upper limit of the $g f$ value of $\lambda 4019.1$ is $0.63(B F=1)$ based on the measured lifetime, whereas the formula gives an adjusted $g f$-value of 0.79 .

In the present paper we present $g f$-values for $180 \mathrm{Th}$ II lines in the wavelength region $3100-12600 \AA$, derived by combining new measurements of both branching fractions $(B F \mathrm{~s})$ and radiative lifetimes. For 13 levels we have used the lifetimes measured by Simonsen et al. (1988). The line identifications are taken from the term analysis by Palmer \& Engleman (1983).

\section{Experiment}

\subsection{Lifetime measurements}

In the present investigation radiative lifetimes of ten levels in Th II were measured using the laser-inducedfluorescence (LIF) technique. A plasma of thorium ions was created by irradiating a thorium target with laser pulses. The measurements were performed close to the target during the expansion of the plasma, in which lowlying metastable levels were populated. The ions were selectively photo-excited to the level under investigation by the light from a pulsed laser system. A Nd:YAG laser pumped a tuneable dye laser, working on a red dye. 
Table 1. Experimental radiative lifetimes of Th II.

\begin{tabular}{|c|c|c|c|c|c|c|c|}
\hline \multirow[t]{2}{*}{ Configuration $^{a}$} & \multirow{2}{*}{$\begin{array}{l}\text { Energy } \\
\left(\mathrm{cm}^{-1}\right)\end{array}$} & \multirow[t]{2}{*}{$J$} & \multirow{2}{*}{$\begin{array}{l}\lambda_{\mathrm{exc}}^{b} \\
(\AA)\end{array}$} & \multirow{2}{*}{$\begin{array}{l}\lambda_{\mathrm{obs}}^{c} \\
(\AA)\end{array}$} & \multicolumn{3}{|c|}{ Exp. Lifetime (ns) ${ }^{d}$} \\
\hline & & & & & this work & $\mathrm{S}$ & $\mathrm{A} \& \mathrm{P}$ \\
\hline $5 f\left({ }^{2} \mathrm{~F}\right) 6 \mathrm{~d}^{2}\left({ }^{3} \mathrm{~F}\right)$ & 18214.426 & 1.5 & & & & $376(38)$ & \\
\hline $5 f\left({ }^{2} F\right) 6 d^{2}\left({ }^{3} F\right)$ & 19050.829 & 1.5 & & & & $579(58)$ & \\
\hline $5 \mathrm{f} 6 \mathrm{~d}\left({ }^{3} \mathrm{D}\right) 7 \mathrm{~s}$ & 19248.270 & 2.5 & & & & $453(45)$ & \\
\hline $5 f\left({ }^{2} \mathrm{~F}\right) 6 \mathrm{~d}^{2}\left({ }^{3} \mathrm{~F}\right)$ & 20686.146 & 2.5 & & & & $502(50)$ & \\
\hline $5 \mathrm{f} 6 \mathrm{~d}\left({ }^{1} \mathrm{P}\right) 7 \mathrm{~s}$ & 21131.799 & 1.5 & & & & $1290(323)$ & \\
\hline $5 \mathrm{f}\left({ }^{2} \mathrm{~F}\right) 6 \mathrm{~d}^{2}\left({ }^{3} \mathrm{~F}\right)$ & 21297.416 & 2.5 & & & & $315(32)$ & \\
\hline $5 \mathrm{f}\left({ }^{2} \mathrm{~F}\right) 6 \mathrm{~d}^{2}\left({ }^{3} \mathrm{~F}\right)$ & 21682.747 & 3.5 & & & & $807(202)$ & \\
\hline $5 \mathrm{f} 6 \mathrm{~d}\left({ }^{1} \mathrm{H}\right) 7 \mathrm{~s}$ & 22642.105 & 4.5 & & & & $3560(890)$ & \\
\hline $6 \mathrm{~d} 7 \mathrm{~s}\left({ }^{3} \mathrm{D}\right) 7 \mathrm{p}$ & 23372.581 & 1.5 & 4277.314 & 4277.314 & $81(5)$ & & \\
\hline $5 \mathrm{f}\left({ }^{2} \mathrm{~F}\right) 6 \mathrm{~d}^{2}\left({ }^{3} \mathrm{~F}\right)$ & 24132.035 & 1.5 & & & & $159(8)$ & \\
\hline $5 f\left({ }^{2} F\right) 6 d^{2}\left({ }^{3} F\right)$ & 24414.641 & 1.5 & & & & $62.6(1.9)$ & \\
\hline $5 \mathrm{f}\left({ }^{2} \mathrm{~F}\right) 6 \mathrm{~d}^{2}\left({ }^{3} \mathrm{~F}\right)$ & 24463.789 & 2.5 & 4086.521 & 4086.521 & $95(6)$ & $94.2(3.0)$ & \\
\hline $6 \mathrm{~d} 7 \mathrm{~s}\left({ }^{3} \mathrm{D}\right) 7 \mathrm{p}$ & 24873.983 & 2.5 & & & & $23.0(7)$ & $21(3)$ \\
\hline $5 \mathrm{f}\left({ }^{2} \mathrm{~F}\right) 6 \mathrm{~d}^{2}\left({ }^{3} \mathrm{P}\right)$ & 25188.120 & 1.5 & & & & $280(28)$ & \\
\hline $5 \mathrm{f}\left({ }^{2} \mathrm{~F}\right) 6 \mathrm{~d}^{2}\left({ }^{3} \mathrm{P}\right)$ & 25440.231 & 2.5 & 4179.715 & 3929.669 & $66(4)$ & $66.2(2.0)$ & \\
\hline $5 \mathrm{f}\left({ }^{2} \mathrm{~F}\right) 6 \mathrm{~d}^{2}\left({ }^{3} \mathrm{P}\right)$ & 26424.470 & 2.5 & 3783.296 & 3783.296 & $140(9)$ & $151(8)$ & \\
\hline $5 \mathrm{f}\left({ }^{2} \mathrm{~F}\right) 6 \mathrm{~d}^{2}\left({ }^{3} \mathrm{P}\right)$ & 27249.544 & 3.5 & & & & $125(6)$ & \\
\hline $6 \mathrm{~d} 7 \mathrm{~s}\left({ }^{3} \mathrm{D}\right) 7 \mathrm{p}$ & 28243.812 & 2.5 & 3539.587 & 3741.183 & $11.5(7)$ & & \\
\hline $6 \mathrm{~d} 7 \mathrm{~s}\left({ }^{3} \mathrm{D}\right) 7 \mathrm{p}$ & 28720.835 & 1.5 & 3675.567 & 3721.825 & $12.5(7)$ & $15.3(5)^{e}$ & \\
\hline $5 \mathrm{f}\left({ }^{2} \mathrm{~F}\right) 6 \mathrm{~d}^{2}\left({ }^{1} \mathrm{D}\right)$ & 29095.464 & 2.5 & 3625.628 & 3435.977 & $28(2)$ & $29(1)$ & \\
\hline $6 \mathrm{~d}^{2}\left({ }^{3} \mathrm{~F}\right) 7 \mathrm{p}$ & 30972.162 & 2.5 & 3227.774 & 3433.999 & $15.5(7)$ & & \\
\hline $6 \mathrm{~d} 7 \mathrm{~s}\left({ }^{3} \mathrm{D}\right) 7 \mathrm{p}$ & 31353.125 & 1.5 & 3351.228 & 3351.228 & $8.8(4)$ & & \\
\hline $6 d^{2}\left({ }^{3} F\right) 7 p$ & 32957.429 & 3.5 & 3180.194 & 3469.924 & $6.5(3)$ & & \\
\hline
\end{tabular}

${ }^{a}$ Notation according to Blaise \& Wyart (1992).

${ }^{b}$ Laser wavelength used to populate the upper state.

${ }^{c}$ Wavelength used to detect the fluorescence signal.

${ }^{d} \mathrm{~S}=$ Simonsen et al. (1988) (Beam-laser), A\&P = Andersen \& Petkov (1975) (Beam-foil).

${ }^{e}$ See discussion in the lifetime section.

The dye laser light was shifted to the desired wavelengths in near UV and deep blue using a frequency-doubling crystal and Raman shifting in a hydrogen cell. Fluorescence light released at the decay of the excited levels was captured using a fast detection system. For levels with short lifetimes the temporal shape of the laser pulse was also recorded, and the lifetime values were evaluated by fitting a convolution of the recorded pulse and an exponential to the fluorescence signal. The experimental set-up is described in detail by Li et al. (2000).

For elements having level and line rich spectra selectivity in both excitation and detection is especially important. The band width of the laser light utilized in the present investigation was about $0.1 \AA$, and the fluorescent light was selected by a monochromator. After setting the laser to the excitation wavelength for a level and observing fluorescence at an expected wavelength, a further test of proper level identification was made by tuning the monochromator to a different decay channel. The lines used for excitation and recording of decay curves are given in Table 1 together with the lifetime values. The excitation wavelengths were taken from Palmer \& Engleman (1983). The advantage with a laser produced plasma as ion source is the high ion density, fairly high population in metastable levels and the presence of high ionization stages. A major drawback is the high speed of the created ions, which for singly ionized thorium is in the range $10^{3}-10^{4} \mathrm{~m} \mathrm{~s}^{-1}$. It sets an upper limit for the lifetime that can be measured due to flight-out-of-view effects (Sikström et al. 2001). 
Possible systematic errors due to flight-out-of-view effects were checked by changing the position of the detecting monochromator slit. Another test was to make lifetime recordings for different delays between the ablation laser pulse and the excitation laser pulse, i.e. to perform measurements on ions with different velocities. Our uncertainties are to equal parts given by statistical scattering between different recordings and possible systematic effects.

Five of the levels now studied were included in the beam-laser work of Simonsen et al. (1988). As can be seen in Table 1 the agreement is good for all lifetimes except the shortest one. The energy of this level is given as $28720.315 \mathrm{~cm}^{-1}$ in the work of Simonsen et al. (1988), but there is no known level in Th II at this energy (Blaise \& Wyart 1992). The closest level is reported at $28720.835 \mathrm{~cm}^{-1}$, but there is also one at $29720.315 \mathrm{~cm}^{-1}$, implying a typo in the table of Simonsen et al. (1988). We measured the lifetimes of both these levels and found the lifetime of 28720 level to be slightly shorter (12.5 ns) than the value (15.3 ns) reported by Simonsen et al. (1988), and the lifetime of the 29720 level to be about $70 \mathrm{~ns}$. Our conclusion is that Simonsen et al. (1988) measured the lifetime of the level at $28720.835 \mathrm{~cm}^{-1}$, and that the deviation from our value might be due to limitations in their measurements.

\subsection{Branching fractions}

Line intensities have been measured in $\mathrm{HC}$ spectra between 2500 and $7000 \AA$ recorded with a Chelsea Instrument FT500 vacuum ultraviolet Fourier transform spectrometer (FTS). The cathode is a $3 \mathrm{~cm}$ long cylinder of iron with an inner diameter of $0.5 \mathrm{~cm}$, and the inner wall is covered with a thorium foil. Spectra were recorded at discharge currents between 0.1 and $0.6 \mathrm{~A}$ using argon as a carrier gas at a pressure of 0.7 Torr.

The intensities of lines above $7000 \AA$ were obtained from spectra recorded with the FTS at Kitt Peak National Observatory (Palmer \& Engleman 1983).

In order to get absolute intensities the spectra had to be corrected for the instrument response. This was done with a tungsten ribbon lamp with known spectral distribution (4100-7000 $\mathrm{A}$ ) and Ar II lines with known branching ratios (BR) (2500-5300 $)$ ) (Whaling et al. 1993). For some Th II levels spectra from different recordings had to be combined, and that produces larger uncertainties in the calibration. The uncertainty of the tungsten lamp calibration data is $3 \%$, and the recorded spectrum from the lamp is reproducible within $5 \%$. The uncertainty in the calibration with known BRs of argon is estimated to $10 \%$, and the uncertainty caused by the combination of different spectral regions is estimated to $10 \%$. The spectra from Kitt Peak were intensity calibrated with a tungsten lamp and an argon mini-arc. Palmer \& Engleman (1983) claim an uncertainty of $5 \%$ in the calibration between

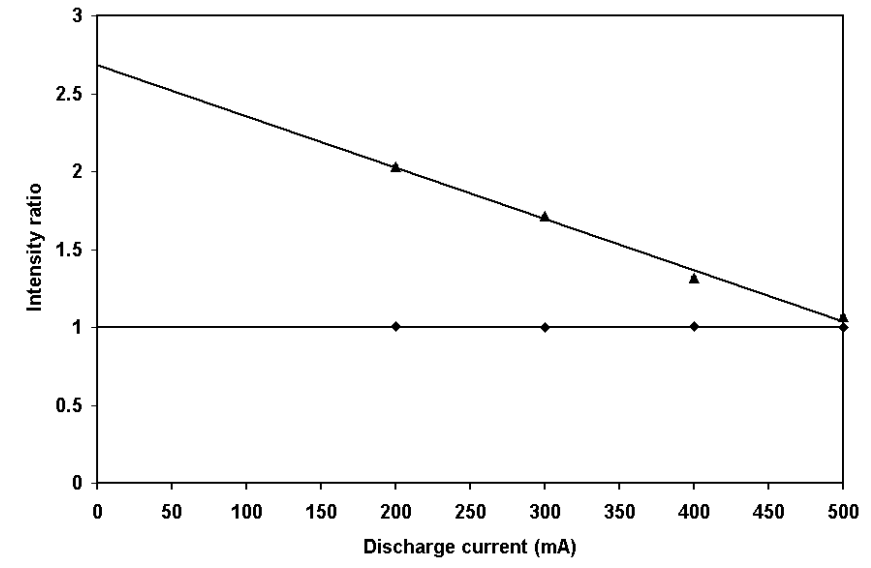

Fig. 1. Self absorption plot for three lines coming from the Th II level at $24873.983 \mathrm{~cm}^{-1}$. The tilted line shows the ratio between the intensities of the strongest line and the two weaker lines, while the horizontal curve shows the ratio between the two weak lines.

4000-13500 ̊, and 10\% when combining different spectral regions.

Strong lines involving the ground state are potentially affected by self absorbtion (SA). In order to check for this effect, the intensity ratio between different lines from the same upper level is plotted against the discharge current through the HC lamp. This is illustrated in Fig. 1, where the intensity relation between three lines coming from the Th II level at $24873.893 \mathrm{~cm}^{-1}$ is plotted. Two of the lines are weak, $B F=0.03$ and 0.01 , respectively, while the third line is strong and involves the ground state. The strong line is clearly affected by SA, since the ratio between this line and the two weaker ones decreases at higher currents, while the ratio between the two weak lines is independent of the current. We adopted the ratio obtained by linear extrapolation to zero current.

\section{Oscillator strengths}

By combining the radiative lifetime $(\tau)$ and $B F$ s one can derive transition probabilities ( $A$-values) for lines from a particular level through the relation

$A=\frac{B F}{\tau}$.

$A$-values can be converted to oscillator strengths or $g f$ values by

$g_{1} f=1.4992 \times 10^{-16} \lambda^{2} g_{\mathrm{u}} A$,

where $\lambda$ is in $\AA, A$ is in $\mathrm{s}^{-1}$, and $g_{\mathrm{u}}$ and $g_{\mathrm{l}}$ are the statistical weights for the upper and lower levels, respectively.

The measured $B F \mathrm{~s}, g f$ and $\log g f$-values of the lines are reported in Table 2. Our log $g f$-values are also compared with the values of Corliss \& Bozman (1962) and Corliss (1979). The total uncertainties in the $g f$-values reported in Table 2 are derived according to the method suggested by Sikström et al. (2001). The included contributions to the uncertainty come from: the intensity measurements, the intensity calibration, combining different 
Table 2. Th II branching fractions $(B F \mathrm{~s})$ and $g f$-values. The lines are sorted by the upper level.

\begin{tabular}{|c|c|c|c|c|c|c|c|c|c|}
\hline \multirow{2}{*}{$\begin{array}{l}\text { Upper } \\
\text { level }^{a}\left(\mathrm{~cm}^{-1}\right)\end{array}$} & \multirow{2}{*}{$\begin{array}{c}\text { Lower } \\
\text { level }\left(\mathrm{cm}^{-1}\right)\end{array}$} & \multirow{2}{*}{$\begin{array}{l}\lambda_{\text {air }} \\
(\AA) \\
\end{array}$} & \multirow{2}{*}{$\begin{array}{c}\sigma \\
\left(\mathrm{cm}^{-1}\right) \\
\end{array}$} & \multirow[t]{2}{*}{$B F$} & \multirow[t]{2}{*}{$g f$} & \multicolumn{3}{|c|}{$\log g f$} & \multirow{2}{*}{$\begin{array}{r}\text { Unc } \\
(\% \text { in } g f) \\
\end{array}$} \\
\hline & & & & & & This work & Corliss $^{b}$ & $\mathrm{C} \& \mathrm{~B}^{c}$ & \\
\hline \multirow{4}{*}{$\begin{array}{l}18214.426 \\
\tau=376 \mathrm{~ns} \\
J=1.5\end{array}$} & 8605.841 & 10404.508 & 9754.074 & $0.03^{d}$ & 0.005 & -2.311 & & & 51 \\
\hline & 1859.938 & 6112.837 & 16354.488 & 0.25 & 0.015 & -1.832 & & -2.942 & 40 \\
\hline & 1521.896 & 5989.045 & 16692.530 & 0.67 & 0.038 & -1.414 & -1.96 & -2.641 & 10 \\
\hline & 0.000 & 5488.629 & 18214.426 & 0.05 & 0.002 & -2.607 & & -3.304 & 13 \\
\hline \multirow{4}{*}{$\begin{array}{l}19050.829 \\
\tau=579 \mathrm{~ns} \\
J=1.5\end{array}$} & 9400.964 & 10360.000 & 9649.865 & $0.06^{d}$ & 0.007 & -2.143 & & & 49 \\
\hline & 4113.359 & 6692.726 & 14937.470 & 0.08 & 0.004 & -2.449 & & -3.420 & 15 \\
\hline & 1859.938 & 5815.422 & 17190.891 & 0.36 & 0.006 & -1.901 & & -3.228 & 12 \\
\hline & 0.000 & 5247.655 & 19050.829 & 0.50 & 0.008 & -1.846 & -2.01 & -2.662 & 11 \\
\hline \multirow{10}{*}{$\begin{array}{l}19248.270 \\
\tau=453 \mathrm{~ns} \\
J=2.5\end{array}$} & 10855.323 & 11911.506 & 8392.947 & $0.02^{d}$ & 0.005 & -2.287 & & & 51 \\
\hline & 9711.962 & 10483.366 & 9536.308 & $0.02^{d}$ & 0.004 & -2.356 & & & 51 \\
\hline & 8605.841 & 9393.774 & 10642.429 & $0.004^{d}$ & 0.001 & -3.158 & & & 51 \\
\hline & 8460.352 & 9267.086 & 10787.918 & $0.03^{d}$ & 0.006 & -2.237 & & & 48 \\
\hline & 7001.420 & 8163.120 & 12246.850 & $0.05^{d}$ & 0.007 & -2.149 & & -3.040 & 45 \\
\hline & 4146.576 & 6619.945 & 15101.694 & 0.18 & 0.015 & -1.812 & & -2.933 & 11 \\
\hline & 4113.359 & 6605.416 & 15134.911 & 0.13 & 0.011 & -1.953 & & -2.936 & 12 \\
\hline & 1859.938 & 5749.388 & 17388.332 & 0.12 & 0.010 & -1.984 & & -3.020 & 11 \\
\hline & 1521.896 & 5639.746 & 17726.374 & 0.38 & 0.025 & -1.605 & -2.03 & -2.677 & 11 \\
\hline & 0.000 & 5193.826 & 19248.270 & 0.07 & 0.004 & -2.380 & & -3.027 & 12 \\
\hline \multirow{10}{*}{$\begin{array}{l}20686.146 \\
\tau=502 \mathrm{~ns} \\
J=2.5\end{array}$} & 10855.323 & 10169.301 & 9830.823 & $0.01^{d}$ & 0.002 & -2.714 & & & 14 \\
\hline & 9711.962 & 9109.794 & 10974.184 & $0.01^{d}$ & 0.001 & -2.989 & & & 14 \\
\hline & 9061.103 & 8599.756 & 11625.043 & $0.02^{d}$ & 0.002 & -2.648 & & & 20 \\
\hline & 8460.352 & 8177.179 & 12225.794 & $0.01^{d}$ & 0.001 & -2.875 & & & 16 \\
\hline & 7001.420 & 7305.404 & 13684.726 & $0.10^{d}$ & 0.010 & -2.023 & & -2.967 & 24 \\
\hline & 4146.576 & 6044.433 & 16539.570 & 0.21 & 0.014 & -1.860 & & -2.875 & 11 \\
\hline & 4113.359 & 6032.318 & 16572.787 & 0.05 & 0.003 & -2.532 & & & 12 \\
\hline & 1859.938 & 5310.267 & 18826.208 & 0.14 & 0.007 & -2.149 & & -2.822 & 11 \\
\hline & 1521.896 & 5216.597 & 19164.250 & 0.19 & 0.009 & -2.039 & & -2.470 & 11 \\
\hline & 0.000 & 4832.803 & 20686.146 & 0.27 & 0.011 & -1.944 & & -2.734 & 11 \\
\hline \multirow{11}{*}{$\begin{array}{l}21131.799 \\
\tau=1290 \mathrm{~ns} \\
J=1.5\end{array}$} & 13250.508 & 12684.806 & 7881.291 & $0.05^{d}$ & 0.004 & -2.433 & & & 55 \\
\hline & 9061.103 & 8282.250 & 12070.696 & $0.05^{d}$ & 0.001 & -2.840 & & & 55 \\
\hline & 8605.841 & 7981.226 & 12525.958 & $0.14^{d}$ & 0.004 & -2.390 & & -3.140 & 50 \\
\hline & 8018.192 & 7623.568 & 13113.607 & $0.04^{d}$ & 0.001 & -2.996 & & & 55 \\
\hline & 7828.559 & 7514.896 & 13303.240 & $0.04^{d}$ & 0.001 & -3.003 & & & 55 \\
\hline & 7001.420 & 7075.000 & 14130.379 & $0.06^{d}$ & 0.001 & -2.846 & & & 54 \\
\hline & 6244.294 & 6715.188 & 14887.505 & 0.08 & 0.002 & -2.767 & & & 29 \\
\hline & 4113.359 & 5874.351 & 17018.440 & 0.10 & 0.002 & -2.814 & & & 27 \\
\hline & 1859.938 & 5187.468 & 19271.861 & 0.11 & 0.001 & -2.855 & & & 27 \\
\hline & 1521.896 & 5098.043 & 19609.903 & 0.29 & 0.004 & -2.451 & & -2.724 & 27 \\
\hline & 0.000 & 4730.881 & 21131.799 & 0.05 & 0.001 & -3.287 & & & 27 \\
\hline \multirow{10}{*}{$\begin{array}{l}21297.416 \\
\tau=315 \mathrm{~ns} \\
J=2.5\end{array}$} & 9711.962 & 8629.143 & 11585.454 & $0.02^{d}$ & 0.003 & -2.487 & & & 51 \\
\hline & 9400.964 & 8403.558 & 11896.452 & $0.02^{d}$ & 0.005 & -2.317 & & & 50 \\
\hline & 8605.841 & 7877.075 & 12691.575 & $0.02^{d}$ & 0.004 & -2.443 & & & 50 \\
\hline & 8460.352 & 7787.800 & 12837.064 & $0.09^{d}$ & 0.016 & -1.810 & & -2.574 & 12 \\
\hline & 8018.192 & 7528.487 & 13279.224 & $0.03^{d}$ & 0.004 & -2.360 & & & 19 \\
\hline & 7001.420 & 6993.037 & 14295.996 & 0.19 & 0.027 & -1.572 & & -2.611 & 11 \\
\hline & 4113.359 & 5817.734 & 17184.057 & 0.02 & 0.002 & -2.785 & & & 12 \\
\hline & 1859.938 & 5143.267 & 19437.478 & 0.15 & 0.012 & -1.933 & & -2.413 & 11 \\
\hline & 1521.896 & 5055.347 & 19775.520 & 0.16 & 0.012 & -1.923 & & -2.484 & 11 \\
\hline & 0.000 & 4694.092 & 21297.416 & 0.30 & 0.019 & -1.728 & & -2.364 & 11 \\
\hline
\end{tabular}


Table 2. continued.

\begin{tabular}{|c|c|c|c|c|c|c|c|c|c|}
\hline \multirow{2}{*}{$\begin{array}{l}\text { Upper } \\
\text { level }^{a}\left(\mathrm{~cm}^{-1}\right)\end{array}$} & \multirow{2}{*}{$\begin{array}{c}\text { Lower } \\
\text { level }\left(\mathrm{cm}^{-1}\right)\end{array}$} & \multirow{2}{*}{$\begin{array}{l}\lambda_{\text {air }} \\
(\AA)\end{array}$} & \multirow{2}{*}{$\begin{array}{c}\sigma \\
\left(\mathrm{cm}^{-1}\right)\end{array}$} & \multirow[t]{2}{*}{$B F$} & \multirow[t]{2}{*}{$g f$} & \multicolumn{3}{|c|}{$\log g f$} & \multirow{2}{*}{$\begin{array}{r}\text { Unc } \\
(\% \text { in } g f) \\
\end{array}$} \\
\hline & & & & & & This work & Corliss $^{b}$ & $C \& B^{c}$ & \\
\hline \multirow{6}{*}{$\begin{array}{l}21682.747 \\
\tau=807 \mathrm{~ns} \\
J=3.5\end{array}$} & 10855.323 & 9233.273 & 10827.424 & $0.02^{d}$ & 0.003 & -2.525 & & & 56 \\
\hline & 9400.964 & 8139.902 & 12281.783 & $0.05^{d}$ & 0.005 & -2.305 & & -3.047 & 54 \\
\hline & 8605.841 & 7644.964 & 13076.906 & $0.02^{d}$ & 0.002 & -2.706 & & & 56 \\
\hline & 6213.490 & 6462.648 & 15469.257 & 0.36 & 0.022 & -1.654 & & & 26 \\
\hline & 4146.576 & 5700.917 & 17536.171 & 0.53 & 0.026 & -1.592 & & -2.240 & 25 \\
\hline & 1521.896 & 4958.724 & 20160.851 & 0.02 & 0.001 & -3.227 & & & 26 \\
\hline \multirow{4}{*}{$\begin{array}{l}22642.105 \\
\tau=3560 \mathrm{~ns} \\
J=4.5\end{array}$} & 10379.122 & 8152.381 & 12262.983 & $0.16^{d}$ & 0.005 & -2.338 & & -2.927 & 49 \\
\hline & 9711.962 & 7731.738 & 12930.143 & $0.45^{d}$ & 0.011 & -1.946 & & -2.695 & 27 \\
\hline & 6213.490 & 6085.256 & 16428.615 & 0.34 & 0.005 & -2.272 & & -2.928 & 27 \\
\hline & 4146.576 & 5405.209 & 18495.529 & 0.04 & 0.001 & -3.274 & & & 28 \\
\hline \multirow{7}{*}{$\begin{array}{l}23372.581 \\
\tau=81 \mathrm{~ns} \\
J=1.5\end{array}$} & 13250.508 & 9876.691 & 10122.073 & $0.004^{d}$ & 0.009 & -2.044 & & & 54 \\
\hline & 12219.976 & 8964.047 & 11152.614 & $0.01^{d}$ & 0.004 & -2.367 & & & 54 \\
\hline & 9061.103 & 6985.472 & 14311.478 & $0.02^{d}$ & 0.007 & -2.151 & & & 53 \\
\hline & 8605.841 & 6770.107 & 14766.740 & 0.05 & 0.005 & -2.269 & & -2.779 & 19 \\
\hline & 8018.192 & 6510.997 & 15354.389 & 0.02 & 0.004 & -2.449 & & & 53 \\
\hline & 4113.359 & 5190.872 & 19259.222 & 0.09 & 0.009 & -2.056 & & -2.426 & 19 \\
\hline & 1521.896 & 4575.233 & 21850.685 & 0.01 & 0.001 & -3.076 & & -2.967 & 19 \\
\hline $\mathrm{SA}$ & 0.000 & 4277.314 & 23372.581 & 0.80 & 0.050 & -1.301 & -1.19 & -1.696 & 7 \\
\hline \multirow{8}{*}{$\begin{array}{l}24132.035 \\
\tau=159 \mathrm{~ns} \\
J=1.5\end{array}$} & 14349.388 & 10219.381 & 9782.647 & $0.02^{d}$ & 0.010 & -1.982 & & & 50 \\
\hline & 12219.976 & 8392.541 & 11912.068 & $0.02^{d}$ & 0.005 & -2.305 & & & 51 \\
\hline & 9061.103 & 6633.458 & 15070.932 & $0.04^{d}$ & 0.008 & -2.090 & & & 49 \\
\hline & 8018.192 & 6204.128 & 16113.843 & $0.04^{d}$ & 0.006 & -2.208 & & & 50 \\
\hline & 7828.559 & 6131.964 & 16303.476 & 0.03 & 0.004 & -2.387 & & & 32 \\
\hline & 6244.294 & 5588.869 & 17887.741 & 0.07 & 0.010 & -1.995 & & -2.555 & 10 \\
\hline & 4113.359 & 4993.942 & 20018.676 & 0.01 & 0.001 & -3.014 & & & 13 \\
\hline & 0.000 & 4142.701 & 24132.035 & 0.77 & 0.058 & -1.239 & & -1.928 & 6 \\
\hline \multirow{7}{*}{$\begin{array}{l}24414.641 \\
\tau=62.6 \mathrm{~ns} \\
J=1.5\end{array}$} & 9061.103 & 6511.358 & 15353.538 & 0.02 & 0.009 & -2.038 & & -2.857 & 13 \\
\hline & 8605.841 & 6323.842 & 15808.800 & 0.01 & 0.004 & -2.434 & & & 51 \\
\hline & 8460.352 & 6266.174 & 15954.289 & 0.04 & 0.017 & -1.767 & & -2.710 & 12 \\
\hline & 8018.192 & 6097.194 & 16396.449 & 0.01 & 0.005 & -2.287 & & & 14 \\
\hline & 7001.420 & 5741.170 & 17413.221 & 0.04 & 0.015 & -1.815 & & -2.587 & 12 \\
\hline & 6244.294 & 5501.944 & 18170.347 & 0.03 & 0.007 & -2.129 & & -2.842 & 12 \\
\hline & 4113.359 & 4924.422 & 20301.282 & 0.03 & 0.008 & -2.095 & & -2.651 & 12 \\
\hline SA & 0.000 & 4094.747 & 24414.641 & 0.81 & 0.130 & -0.885 & -0.99 & -1.461 & 4 \\
\hline \multirow{12}{*}{$\begin{array}{l}24463.789 \\
\tau=95 \mathrm{~ns} \\
J=2.5\end{array}$} & 15786.985 & 11521.826 & 8676.804 & $0.01^{d}$ & 0.007 & -2.147 & & & 51 \\
\hline & 15236.637 & 10834.612 & 9227.152 & $0.01^{d}$ & 0.006 & -2.200 & & & 51 \\
\hline & 12219.976 & 8165.139 & 12243.822 & $0.01^{d}$ & 0.005 & -2.314 & & & 51 \\
\hline & 10855.323 & 7346.343 & 13608.466 & $0.01^{d}$ & 0.007 & -2.172 & & -2.840 & 51 \\
\hline & 8605.841 & 6304.243 & 15857.948 & 0.03 & 0.012 & -1.934 & & -2.696 & 11 \\
\hline & 8460.352 & 6246.930 & 16003.437 & 0.01 & 0.003 & -2.526 & & & 28 \\
\hline & 8018.192 & 6078.972 & 16445.597 & 0.01 & 0.004 & -2.399 & & & 26 \\
\hline & 7001.420 & 5725.012 & 17462.369 & 0.02 & 0.007 & -2.152 & & & 11 \\
\hline & 4146.576 & 4920.561 & 20317.213 & 0.02 & 0.004 & -2.417 & & & 10 \\
\hline & 4113.359 & 4912.529 & 20350.430 & 0.08 & 0.018 & -1.732 & & -2.461 & 10 \\
\hline & 1859.938 & 4422.783 & 22603.851 & 0.02 & 0.003 & -2.549 & & & 10 \\
\hline & 1521.896 & 4357.613 & 22941.893 & 0.04 & 0.007 & -2.173 & & -2.479 & 10 \\
\hline $\mathrm{SA}$ & 0.000 & 4086.521 & 24463.789 & 0.74 & 0.118 & -0.929 & -0.99 & -1.458 & 6 \\
\hline
\end{tabular}


Table 2. continued.

\begin{tabular}{|c|c|c|c|c|c|c|c|c|c|}
\hline \multirow{2}{*}{$\begin{array}{l}\text { Upper } \\
\text { level }^{a}\left(\mathrm{~cm}^{-1}\right)\end{array}$} & \multirow{2}{*}{$\begin{array}{c}\text { Lower } \\
\text { level }\left(\mathrm{cm}^{-1}\right)\end{array}$} & \multirow{2}{*}{$\begin{array}{l}\lambda_{\text {air }} \\
(\AA)\end{array}$} & \multirow{2}{*}{$\begin{array}{c}\sigma \\
\left(\mathrm{cm}^{-1}\right)\end{array}$} & \multirow[t]{2}{*}{$B F$} & \multirow[t]{2}{*}{$g f$} & \multicolumn{3}{|c|}{$\log g f$} & \multirow{2}{*}{$\begin{array}{r}\text { Unc. } \\
(\% \text { in } g f)\end{array}$} \\
\hline & & & & & & This work & Corliss $^{b}$ & $\mathrm{C} \& \mathrm{~B}^{c}$ & \\
\hline 24873.983 & 8605.841 & 6145.283 & 16268.142 & 0.004 & 0.006 & -2.244 & & & 21 \\
\hline$\tau=23.0 \mathrm{~ns}$ & 7001.420 & 5593.615 & 17872.563 & 0.01 & 0.016 & -1.782 & & & 21 \\
\hline \multirow[t]{3}{*}{$J=2.5$} & 4146.576 & 4823.182 & 20727.407 & 0.01 & 0.005 & -2.313 & & -2.675 & 21 \\
\hline & 1859.938 & 4343.951 & 23014.045 & 0.01 & 0.011 & -1.958 & & -2.173 & 21 \\
\hline & 1521.896 & 4281.068 & 23352.087 & 0.03 & 0.018 & -1.746 & & -2.078 & 21 \\
\hline $\mathrm{SA}$ & 0.000 & 4019.129 & 24873.983 & 0.94 & 0.592 & -0.228 & -0.19 & -0.651 & 3 \\
\hline 25188.120 & 15236.637 & 10045.999 & 9951.483 & $0.01^{d}$ & 0.003 & -2.521 & & & 51 \\
\hline$\tau=280 \mathrm{~ns}$ & 9400.964 & 6332.512 & 15787.156 & 0.11 & 0.010 & -2.012 & & & 12 \\
\hline \multirow[t]{4}{*}{$J=1.5$} & 8018.192 & 5822.522 & 17169.928 & 0.05 & 0.003 & -2.482 & & & 12 \\
\hline & 4113.359 & 4743.685 & 21074.761 & 0.20 & 0.010 & -2.019 & & -2.367 & 11 \\
\hline & 1521.896 & 4224.241 & 23666.224 & 0.20 & 0.008 & -2.127 & & -2.430 & 11 \\
\hline & 0.000 & 3969.003 & 25188.120 & 0.43 & 0.015 & -1.835 & & -2.407 & 11 \\
\hline 25440.231 & 15786.985 & 10356.371 & 9653.246 & $0.005^{d}$ & 0.007 & -2.163 & & & 51 \\
\hline$\tau=66 \mathrm{~ns}$ & 10855.323 & 6854.511 & 14584.908 & $0.01^{d}$ & 0.006 & -2.205 & & & 51 \\
\hline \multirow[t]{6}{*}{$J=2.5$} & 9400.964 & 6232.974 & 16039.267 & 0.03 & 0.014 & -1.865 & & -2.554 & 12 \\
\hline & 9061.103 & 6103.641 & 16379.128 & 0.02 & 0.011 & -1.970 & & & 12 \\
\hline & 8605.841 & 5938.576 & 16834.390 & 0.02 & 0.011 & -1.964 & & & 12 \\
\hline & 7001.420 & 5421.836 & 18438.811 & 0.03 & 0.011 & -1.946 & & -2.536 & 11 \\
\hline & 4146.576 & 4694.921 & 21293.655 & 0.03 & 0.010 & -1.998 & & -2.303 & 11 \\
\hline & 1521.896 & 4179.715 & 23918.335 & 0.20 & 0.047 & -1.330 & & -1.756 & 11 \\
\hline $\mathrm{SA}$ & 0.000 & 3929.669 & 25440.231 & 0.66 & 0.138 & -0.859 & -1.03 & -1.463 & 7 \\
\hline 26424.470 & 9711.962 & 5981.885 & 16712.508 & 0.04 & 0.012 & -1.920 & & & 49 \\
\hline$\tau=140 \mathrm{~ns}$ & 9400.964 & 5872.603 & 17023.506 & 0.07 & 0.019 & -1.730 & & & 9 \\
\hline \multirow[t]{4}{*}{$J=2.5$} & 4146.576 & 4487.496 & 22277.894 & 0.40 & 0.045 & -1.322 & & -1.899 & 8 \\
\hline & 1859.938 & 4069.761 & 24564.532 & 0.03 & 0.003 & -2.572 & & & 9 \\
\hline & 1521.896 & 4014.514 & 24902.574 & 0.08 & 0.007 & -2.131 & & -2.345 & 9 \\
\hline & 0.000 & 3783.296 & 26424.470 & 0.38 & 0.037 & -1.436 & & -2.017 & 8 \\
\hline 27249.544 & 15786.985 & 8721.660 & 11462.559 & $0.04^{d}$ & 0.027 & -1.525 & & -2.275 & 39 \\
\hline$\tau=125 \mathrm{~ns}$ & 13248.708 & 7140.462 & 14000.836 & $0.04^{d}$ & 0.020 & -1.659 & & -2.535 & 30 \\
\hline \multirow[t]{8}{*}{$J=3.5$} & 12570.493 & 6810.550 & 14679.051 & $0.06^{d}$ & 0.024 & -1.586 & & -2.393 & 28 \\
\hline & 10379.122 & 5925.892 & 16870.422 & 0.16 & 0.058 & -1.260 & & -2.198 & 8 \\
\hline & 9711.962 & 5700.459 & 17537.582 & 0.09 & 0.029 & -1.570 & & -2.148 & 8 \\
\hline & 8605.841 & 5362.250 & 18643.703 & 0.02 & 0.006 & -2.249 & & & 9 \\
\hline & 6213.490 & 4752.414 & 21036.054 & 0.49 & 0.114 & -0.971 & & -1.619 & 6 \\
\hline & 4146.576 & 4327.231 & 23102.969 & 0.04 & 0.007 & -2.152 & & -2.285 & 8 \\
\hline & 4113.359 & 4321.019 & 23136.185 & 0.01 & 0.002 & -2.725 & & & 9 \\
\hline & 1521.896 & 3885.768 & 25727.648 & 0.04 & 0.007 & -2.207 & & -2.287 & 8 \\
\hline 28243.812 & 9400.964 & 5305.577 & 18842.848 & 0.01 & 0.023 & -1.636 & & -2.35 & 14 \\
\hline$\tau=11.5 \mathrm{~ns}$ & 9061.103 & 5211.577 & 19182.709 & 0.002 & 0.003 & -2.477 & & & 51 \\
\hline \multirow[t]{5}{*}{$J=2.5$} & 8018.192 & 4942.844 & 20225.620 & 0.003 & 0.006 & -2.187 & & & 15 \\
\hline & 7001.420 & 4706.251 & 21242.392 & 0.02 & 0.032 & -1.490 & & -2.18 & 12 \\
\hline & 4146.576 & 4148.684 & 24097.236 & 0.003 & 0.004 & -2.387 & & & 15 \\
\hline & 4113.359 & 4142.973 & 24130.453 & 0.003 & 0.004 & -2.429 & & & 15 \\
\hline & 1859.938 & 3789.118 & 26383.874 & 0.05 & 0.055 & -1.259 & -1.10 & -1.60 & 11 \\
\hline $\mathrm{SA}$ & 1521.896 & 3741.183 & 26721.916 & 0.62 & 0.677 & -0.170 & -0.50 & -1.00 & 8 \\
\hline $\mathrm{SA}$ & 0.000 & 3539.587 & 28243.812 & 0.29 & 0.287 & -0.542 & -0.86 & -1.35 & 13 \\
\hline
\end{tabular}


Table 2. continued.

\begin{tabular}{|c|c|c|c|c|c|c|c|c|c|}
\hline \multirow{2}{*}{$\begin{array}{l}\text { Upper } \\
\text { level }^{a}\left(\mathrm{~cm}^{-1}\right)\end{array}$} & \multirow{2}{*}{$\begin{array}{c}\text { Lower } \\
\text { level }\left(\mathrm{cm}^{-1}\right)\end{array}$} & \multirow{2}{*}{$\begin{array}{l}\lambda_{\text {air }} \\
(\AA)\end{array}$} & \multirow{2}{*}{$\begin{array}{c}\sigma \\
\left(\mathrm{cm}^{-1}\right)\end{array}$} & \multirow[t]{2}{*}{$B F$} & \multirow[t]{2}{*}{$g f$} & \multicolumn{3}{|c|}{$\log g f$} & \multirow{2}{*}{$\begin{array}{r}\text { Unc. } \\
(\% \text { in } g f)\end{array}$} \\
\hline & & & & & & This work & Corliss $^{b}$ & $\mathrm{C} \& \mathrm{~B}^{c}$ & \\
\hline 28720.835 & 12219.976 & 6058.610 & 16500.868 & 0.01 & 0.015 & -1.823 & & & 51 \\
\hline$\tau=15.3 \mathrm{~ns}$ & 8605.841 & 4970.029 & 20114.994 & 0.02 & 0.023 & -1.647 & & -1.924 & 11 \\
\hline \multirow[t]{2}{*}{$J=1.5$} & 7001.420 & 4602.886 & 21719.415 & 0.03 & 0.027 & -1.576 & & -1.973 & 11 \\
\hline & 6244.294 & 4447.834 & 22476.541 & 0.06 & 0.059 & -1.232 & & -1.629 & 11 \\
\hline SA & 1859.938 & 3721.825 & 26860.897 & 0.61 & 0.406 & -0.391 & -0.69 & -1.016 & 8 \\
\hline SA & 1521.896 & 3675.567 & 27198.939 & 0.27 & 0.177 & -0.752 & -0.75 & -1.074 & 13 \\
\hline 29095.464 & 18118.701 & 9107.654 & 10976.763 & $0.01^{d}$ & 0.014 & -1.871 & & & 51 \\
\hline$\tau=28 \mathrm{~ns}$ & 12570.493 & 6049.773 & 16524.971 & 0.01 & 0.010 & -1.991 & & & 50 \\
\hline \multirow[t]{8}{*}{$J=2.5$} & 10855.323 & 5480.863 & 18240.141 & 0.01 & 0.013 & -1.872 & & & 10 \\
\hline & 9061.103 & 4990.032 & 20034.361 & 0.01 & 0.008 & -2.100 & & & 10 \\
\hline & 8605.841 & 4879.156 & 20489.623 & 0.01 & 0.008 & -2.079 & & & 10 \\
\hline & 8460.352 & 4844.755 & 20635.112 & 0.02 & 0.012 & -1.940 & & & 10 \\
\hline & 7001.420 & 4524.838 & 22094.044 & 0.03 & 0.021 & -1.681 & & -2.001 & 9 \\
\hline & 4146.576 & 4007.062 & 24948.888 & 0.01 & 0.007 & -2.153 & & & 10 \\
\hline & 4113.359 & 4001.734 & 24982.105 & 0.03 & 0.014 & -1.865 & & -2.022 & 9 \\
\hline & 1521.896 & 3625.628 & 27573.568 & 0.28 & 0.112 & -0.950 & -0.99 & -1.300 & 9 \\
\hline SA & 0.000 & 3435.977 & 29095.464 & 0.58 & 0.214 & -0.670 & -0.76 & -1.075 & 6 \\
\hline 30972.162 & 9711.962 & 4702.309 & 21260.200 & 0.01 & 0.014 & -1.850 & & -2.146 & 8 \\
\hline$\tau=15.5 \mathrm{~ns}$ & 8460.352 & 4440.866 & 22511.810 & 0.11 & 0.120 & -0.919 & & -1.251 & 8 \\
\hline \multirow[t]{3}{*}{$J=2.5$} & 8018.192 & 4355.321 & 22953.970 & 0.05 & 0.056 & -1.253 & & -1.381 & 8 \\
\hline & 4146.576 & 3726.724 & 26825.586 & 0.11 & 0.088 & -1.055 & & -1.283 & 8 \\
\hline & 4113.359 & 3722.115 & 26858.803 & 0.25 & 0.204 & -0.691 & -0.84 & -1.096 & 8 \\
\hline \multirow[t]{3}{*}{ SA } & 1859.938 & 3433.999 & 29112.224 & 0.42 & 0.290 & -0.537 & -0.49 & -0.741 & 8 \\
\hline & 1521.896 & 3394.581 & 29450.266 & 0.005 & 0.003 & -2.497 & & & 17 \\
\hline & 0.000 & 3227.774 & 30972.162 & 0.04 & 0.025 & -1.597 & & -1.771 & 9 \\
\hline 31353.125 & 8605.841 & 4394.895 & 22747.284 & 0.04 & 0.030 & -1.529 & & -1.434 & 10 \\
\hline$\tau=8.8 \mathrm{~ns}$ & 7828.559 & 4249.679 & 23524.566 & 0.04 & 0.026 & -1.583 & & -1.667 & 10 \\
\hline \multirow[t]{3}{*}{$J=1.5$} & 7001.420 & 4105.330 & 24351.705 & 0.12 & 0.071 & -1.148 & & -1.021 & 10 \\
\hline & 4113.359 & 3670.058 & 27239.766 & 0.05 & 0.030 & -1.517 & & -1.150 & 10 \\
\hline & 1859.938 & 3389.640 & 29493.187 & 0.19 & 0.082 & -1.088 & -0.97 & -1.211 & 9 \\
\hline \multirow[t]{2}{*}{$\mathrm{SA}$} & 1521.896 & 3351.228 & 29831.229 & 0.55 & 0.251 & -0.600 & -0.49 & -0.726 & 7 \\
\hline & 0.000 & 3188.553 & 31353.125 & $0.01^{d}$ & 0.002 & -2.627 & & & 51 \\
\hline 32957.429 & 9400.964 & 4243.924 & 23556.465 & 0.02 & 0.063 & -1.202 & & -1.283 & 12 \\
\hline$\tau=6.5 \mathrm{~ns}$ & 9061.103 & 4183.565 & 23896.326 & 0.02 & 0.048 & -1.318 & & -1.491 & 10 \\
\hline \multirow[t]{3}{*}{$J=3.5$} & 8605.841 & 4105.350 & 24351.588 & 0.04 & 0.112 & -0.949 & & & 10 \\
\hline & 4146.576 & 3469.921 & 28810.853 & 0.40 & 0.743 & -0.129 & -0.18 & -0.361 & 9 \\
\hline & 4113.359 & 3465.924 & 28844.070 & 0.03 & 0.057 & -1.246 & & -1.363 & 10 \\
\hline SA & 1521.896 & 3180.194 & 31435.533 & 0.49 & 1.064 & +0.027 & -0.36 & -0.547 & 7 \\
\hline
\end{tabular}

${ }^{a} \mathrm{SA}$ indicates that the line has been corrected for self absorption.

${ }^{b}$ Values reported by Corliss (1979).

${ }^{c}$ Values reported by Corliss \& Bozman (1962).

${ }^{d}$ Indicates that the line intensity is taken from Palmer \& Engleman (1983).

spectral regions, the self absorption correction and the lifetime measurements. The uncertainty introduced by the fact that not all decay channels of a level can be observed (either because they are too weak or because they fall outside the covered wavelength interval), is not included but we have considered the residual BF due to such lines. Calculations with the Cowan code (Cowan 1981) were performed of the even configurations $6 \mathrm{~d}^{2} 7 \mathrm{~s}, 6 \mathrm{~d} 7 \mathrm{~s}^{2}, 6 \mathrm{~d}^{3}$, $5 \mathrm{f}^{2} 7 \mathrm{~s}, 5 \mathrm{f} 7 \mathrm{~s} 7 \mathrm{p}$ and $5 \mathrm{f}^{2} 6 \mathrm{~d}$, and the odd configurations $5 \mathrm{f} 7 \mathrm{~s}^{2}$, $5 \mathrm{f} 6 \mathrm{~d} 7 \mathrm{~s}, 5 \mathrm{f}^{2} \mathrm{~d}^{2}, 6 \mathrm{~d} 7 \mathrm{~s} 7 \mathrm{p}$ and $6 \mathrm{~d}^{2} 7 \mathrm{p}$. In these calculations no 
Table 3. Finding list for Th II transitions sorted by wavelength.

\begin{tabular}{|c|c|c|c|c|}
\hline $\begin{array}{l}\lambda_{\text {air }} \\
(\AA)\end{array}$ & $\begin{array}{r}\text { Lower level } \\
\quad\left(\mathrm{cm}^{-1}\right)\end{array}$ & $\begin{array}{r}\text { Upper level } \\
\left(\mathrm{cm}^{-1}\right)\end{array}$ & $\overline{\log g f}$ & Unc. $^{a}$ \\
\hline 3180.194 & 1521.896 & 32957.429 & +0.027 & 7 \\
\hline 3188.553 & 0.000 & 31353.125 & -2.627 & 51 \\
\hline 3227.774 & 0.000 & 30972.162 & -1.597 & 9 \\
\hline 3351.228 & 1521.896 & 31353.125 & -0.600 & 7 \\
\hline 3389.640 & 1859.938 & 31353.125 & -1.088 & 9 \\
\hline 3394.581 & 1521.896 & 30972.162 & -2.497 & 17 \\
\hline 3433.999 & 1859.938 & 30972.162 & -0.537 & 8 \\
\hline 3435.977 & 0.000 & 29095.464 & -0.670 & 6 \\
\hline 3465.924 & 4113.359 & 32957.429 & -1.246 & 10 \\
\hline 3469.921 & 4146.576 & 32957.429 & -0.129 & 9 \\
\hline 3539.587 & 0.000 & 28243.812 & -0.542 & 13 \\
\hline 3625.628 & 1521.896 & 29095.464 & -0.950 & 9 \\
\hline 3670.058 & 4113.359 & 31353.125 & -1.517 & 10 \\
\hline 3675.567 & 1521.896 & 28720.835 & -0.840 & 13 \\
\hline 3721.825 & 1859.938 & 28720.835 & -0.479 & 8 \\
\hline 3722.115 & 4113.359 & 30972.162 & -0.691 & 8 \\
\hline 3726.724 & 4146.576 & 30972.162 & -1.055 & 8 \\
\hline 3741.183 & 1521.896 & 28243.812 & -0.170 & 8 \\
\hline 3783.296 & 0.000 & 26424.470 & -1.436 & 8 \\
\hline 3789.118 & 1859.938 & 28243.812 & -1.259 & 11 \\
\hline 3885.768 & 1521.896 & 27249.544 & -2.207 & 8 \\
\hline 3929.669 & 0.000 & 25440.231 & -0.859 & 7 \\
\hline 3969.003 & 0.000 & 25188.120 & -1.835 & 11 \\
\hline 4001.734 & 4113.359 & 29095.464 & -1.865 & 9 \\
\hline 4007.062 & 4146.576 & 29095.464 & -2.153 & 10 \\
\hline 4014.514 & 1521.896 & 26424.470 & -2.131 & 9 \\
\hline 4019.129 & 0.000 & 24873.983 & -0.228 & 3 \\
\hline 4069.761 & 1859.938 & 26424.470 & -2.572 & 9 \\
\hline 4086.521 & 0.000 & 24463.789 & -0.929 & 6 \\
\hline 4094.747 & 0.000 & 24414.641 & -0.885 & 4 \\
\hline 4105.330 & 7001.420 & 31353.125 & -0.949 & 10 \\
\hline 4105.350 & 8605.841 & 32957.429 & -1.074 & 10 \\
\hline 4142.701 & 0.000 & 24132.035 & -1.239 & 6 \\
\hline 4142.973 & 4113.359 & 28243.812 & -2.429 & 15 \\
\hline 4148.684 & 4146.576 & 28243.812 & -2.387 & 15 \\
\hline 4179.715 & 1521.896 & 25440.231 & -1.330 & 11 \\
\hline 4183.565 & 9061.103 & 32957.429 & -1.318 & 10 \\
\hline 4224.241 & 1521.896 & 25188.120 & -2.127 & 11 \\
\hline 4243.924 & 9400.964 & 32957.429 & -1.202 & 12 \\
\hline 4249.679 & 7828.559 & 31353.125 & -1.583 & 10 \\
\hline 4277.314 & 0.000 & 23372.581 & -1.301 & 7 \\
\hline 4281.068 & 1521.896 & 24873.983 & -1.746 & 21 \\
\hline 4321.019 & 4113.359 & 27249.544 & -2.725 & 9 \\
\hline 4327.231 & 4146.576 & 27249.544 & -2.152 & 8 \\
\hline 4343.951 & 1859.938 & 24873.983 & -1.958 & 21 \\
\hline 4355.321 & 8018.192 & 30972.162 & -1.253 & 8 \\
\hline 4357.613 & 1521.896 & 24463.789 & -2.173 & 10 \\
\hline 4394.895 & 8605.841 & 31353.125 & -1.529 & 10 \\
\hline 4422.783 & 1859.938 & 24463.789 & -2.549 & 10 \\
\hline 4440.866 & 8460.352 & 30972.162 & -0.919 & 8 \\
\hline 4447.834 & 6244.294 & 28720.835 & -1.320 & 11 \\
\hline 4487.496 & 4146.576 & 26424.470 & -1.322 & 8 \\
\hline 4524.838 & 7001.420 & 29095.464 & -1.681 & 9 \\
\hline 4575.233 & 1521.896 & 23372.581 & -3.076 & 19 \\
\hline 4602.886 & 7001.420 & 28720.835 & -1.663 & 11 \\
\hline 4694.092 & 0.000 & 21297.416 & -1.728 & 11 \\
\hline 4694.921 & 4146.576 & 25440.231 & -1.998 & 11 \\
\hline
\end{tabular}

Table 3. continued.

\begin{tabular}{|c|c|c|c|c|}
\hline $\begin{array}{l}\lambda_{\text {air }} \\
(\AA)\end{array}$ & $\begin{array}{r}\text { Lower level } \\
\left(\mathrm{cm}^{-1}\right)\end{array}$ & $\begin{array}{r}\text { Upper level } \\
\left(\mathrm{cm}^{-1}\right)\end{array}$ & 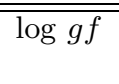 & Unc. $^{a}$ \\
\hline 4702.309 & 9711.962 & 30972.162 & -1.850 & 8 \\
\hline 4706.251 & 7001.420 & 28243.812 & -1.490 & 12 \\
\hline 4730.881 & 0.000 & 21131.799 & -3.287 & 27 \\
\hline 4743.685 & 4113.359 & 25188.120 & -2.019 & 11 \\
\hline 4752.414 & 6213.490 & 27249.544 & -0.971 & 6 \\
\hline 4823.182 & 4146.576 & 24873.983 & -2.313 & 21 \\
\hline 4832.803 & 0.000 & 20686.146 & -1.944 & 11 \\
\hline 4844.755 & 8460.352 & 29095.464 & -1.940 & 10 \\
\hline 4879.156 & 8605.841 & 29095.464 & -2.079 & 10 \\
\hline 4912.529 & 4113.359 & 24463.789 & -1.732 & 10 \\
\hline 4920.561 & 4146.576 & 24463.789 & -2.417 & 10 \\
\hline 4924.422 & 4113.359 & 24414.641 & -2.095 & 12 \\
\hline 4942.844 & 8018.192 & 28243.812 & -2.187 & 15 \\
\hline 4958.724 & 1521.896 & 21682.747 & -3.227 & 26 \\
\hline 4970.029 & 8605.841 & 28720.835 & -1.735 & 11 \\
\hline 4990.032 & 9061.103 & 29095.464 & -2.100 & 10 \\
\hline 4993.942 & 4113.359 & 24132.035 & -3.014 & 13 \\
\hline 5055.347 & 1521.896 & 21297.416 & -1.923 & 11 \\
\hline 5098.043 & 1521.896 & 21131.799 & -2.451 & 27 \\
\hline 5143.267 & 1859.938 & 21297.416 & -1.933 & 11 \\
\hline 5187.468 & 1859.938 & 21131.799 & -2.855 & 27 \\
\hline 5190.872 & 4113.359 & 23372.581 & -2.056 & 19 \\
\hline 5193.826 & 0.000 & 19248.270 & -2.380 & 12 \\
\hline 5211.577 & 9061.103 & 28243.812 & -2.477 & 51 \\
\hline 5216.597 & 1521.896 & 20686.146 & -2.039 & 11 \\
\hline 5247.655 & 0.000 & 19050.829 & -1.846 & 11 \\
\hline 5305.577 & 9400.964 & 28243.812 & -1.636 & 14 \\
\hline 5310.267 & 1859.938 & 20686.146 & -2.149 & 11 \\
\hline 5362.250 & 8605.841 & 27249.544 & -2.249 & 9 \\
\hline 5405.209 & 4146.576 & 22642.105 & -3.274 & 28 \\
\hline 5421.836 & 7001.420 & 25440.231 & -1.946 & 11 \\
\hline 5480.863 & 10855.323 & 29095.464 & -1.872 & 10 \\
\hline 5488.629 & 0.000 & 18214.426 & -2.607 & 13 \\
\hline 5501.944 & 6244.294 & 24414.641 & -2.129 & 12 \\
\hline 5588.869 & 6244.294 & 24132.035 & -1.995 & 10 \\
\hline 5593.615 & 7001.420 & 24873.983 & -1.782 & 21 \\
\hline 5639.746 & 1521.896 & 19248.270 & -1.605 & 11 \\
\hline 5700.459 & 9711.962 & 27249.544 & -1.570 & 8 \\
\hline 5700.917 & 4146.576 & 21682.747 & -1.592 & 25 \\
\hline 5725.012 & 7001.420 & 24463.789 & -2.152 & 11 \\
\hline 5741.170 & 7001.420 & 24414.641 & -1.815 & 12 \\
\hline 5749.388 & 1859.938 & 19248.270 & -1.984 & 11 \\
\hline 5815.422 & 1859.938 & 19050.829 & -1.901 & 12 \\
\hline 5817.734 & 4113.359 & 21297.416 & -2.785 & 12 \\
\hline 5822.522 & 8018.192 & 25188.120 & -2.482 & 12 \\
\hline 5872.603 & 9400.964 & 26424.470 & -1.730 & 9 \\
\hline 5874.351 & 4113.359 & 21131.799 & -2.814 & 27 \\
\hline 5925.892 & 10379.122 & 27249.544 & -1.260 & 8 \\
\hline 5938.576 & 8605.841 & 25440.231 & -1.964 & 12 \\
\hline 5981.885 & 9711.962 & 26424.470 & -1.920 & 49 \\
\hline 5989.045 & 1521.896 & 18214.426 & -1.414 & 10 \\
\hline 6032.318 & 4113.359 & 20686.146 & -2.532 & 12 \\
\hline 6044.433 & 4146.576 & 20686.146 & -1.860 & 11 \\
\hline 6049.773 & 12570.493 & 29095.464 & -1.991 & 50 \\
\hline 6058.610 & 12219.976 & 28720.835 & -1.911 & 51 \\
\hline 6078.972 & 8018.192 & 24463.789 & -2.399 & 26 \\
\hline 6085.256 & 6213.490 & 22642.105 & -2.272 & 27 \\
\hline
\end{tabular}


Table 3. continued.

\begin{tabular}{|c|c|c|c|c|}
\hline $\begin{array}{l}\lambda_{\text {air }} \\
(\AA)\end{array}$ & $\begin{array}{r}\text { Lower level } \\
\left(\mathrm{cm}^{-1}\right)\end{array}$ & $\begin{array}{r}\text { Upper level } \\
\left(\mathrm{cm}^{-1}\right)\end{array}$ & 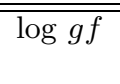 & 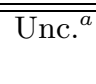 \\
\hline 6097.194 & 8018.192 & 24414.641 & -2.287 & 14 \\
\hline 6103.641 & 9061.103 & 25440.231 & -1.970 & 12 \\
\hline 6112.837 & 1859.938 & 18214.426 & -1.832 & 40 \\
\hline 6131.964 & 7828.559 & 24132.035 & -2.387 & 32 \\
\hline 6145.283 & 8605.841 & 24873.983 & -2.244 & 21 \\
\hline 6204.128 & 8018.192 & 24132.035 & -2.208 & 50 \\
\hline 6232.974 & 9400.964 & 25440.231 & -1.865 & 12 \\
\hline 6246.930 & 8460.352 & 24463.789 & -2.526 & 28 \\
\hline 6266.174 & 8460.352 & 24414.641 & -1.767 & 12 \\
\hline 6304.243 & 8605.841 & 24463.789 & -1.934 & 11 \\
\hline 6323.842 & 8605.841 & 24414.641 & -2.434 & 51 \\
\hline 6332.512 & 9400.964 & 25188.120 & -2.012 & 12 \\
\hline 6462.648 & 6213.490 & 21682.747 & -1.654 & 26 \\
\hline 6510.997 & 8018.192 & 23372.581 & -2.449 & 53 \\
\hline 6511.358 & 9061.103 & 24414.641 & -2.038 & 13 \\
\hline 6605.416 & 4113.359 & 19248.270 & -1.953 & 12 \\
\hline 6619.945 & 4146.576 & 19248.270 & -1.812 & 11 \\
\hline 6633.458 & 9061.103 & 24132.035 & -2.090 & 49 \\
\hline 6692.726 & 4113.359 & 19050.829 & -2.449 & 15 \\
\hline 6715.188 & 6244.294 & 21131.799 & -2.767 & 29 \\
\hline 6770.107 & 8605.841 & 23372.581 & -2.269 & 19 \\
\hline 6810.550 & 12570.493 & 27249.544 & -1.586 & 28 \\
\hline 6854.511 & 10855.323 & 25440.231 & -2.205 & 51 \\
\hline 6985.472 & 9061.103 & 23372.581 & -2.151 & 53 \\
\hline 6993.037 & 7001.420 & 21297.416 & -1.572 & 11 \\
\hline 7075.000 & 7001.420 & 21131.799 & -2.846 & 54 \\
\hline 7140.462 & 13248.708 & 27249.544 & -1.659 & 30 \\
\hline 7305.404 & 7001.420 & 20686.146 & -2.023 & 24 \\
\hline 7346.343 & 10855.323 & 24463.789 & -2.172 & 51 \\
\hline 7514.896 & 7828.559 & 21131.799 & -3.003 & 55 \\
\hline 7528.487 & 8018.192 & 21297.416 & -2.360 & 19 \\
\hline 7623.568 & 8018.192 & 21131.799 & -2.996 & 55 \\
\hline 7644.964 & 8605.841 & 21682.747 & -2.706 & 56 \\
\hline 7731.738 & 9711.962 & 22642.105 & -1.946 & 27 \\
\hline 7787.800 & 8460.352 & 21297.416 & -1.810 & 12 \\
\hline 7877.075 & 8605.841 & 21297.416 & -2.443 & 50 \\
\hline 7981.226 & 8605.841 & 21131.799 & -2.390 & 50 \\
\hline 8139.902 & 9400.964 & 21682.747 & -2.305 & 54 \\
\hline 8152.381 & 10379.122 & 22642.105 & -2.338 & 49 \\
\hline 8163.120 & 7001.420 & 19248.270 & -2.149 & 45 \\
\hline 8165.139 & 12219.976 & 24463.789 & -2.314 & 51 \\
\hline 8177.179 & 8460.352 & 20686.146 & -2.875 & 16 \\
\hline 8282.250 & 9061.103 & 21131.799 & -2.840 & 55 \\
\hline 8392.541 & 12219.976 & 24132.035 & -2.305 & 51 \\
\hline 8403.558 & 9400.964 & 21297.416 & -2.317 & 50 \\
\hline 8599.756 & 9061.103 & 20686.146 & -2.648 & 20 \\
\hline 8629.143 & 9711.962 & 21297.416 & -2.487 & 51 \\
\hline 8721.660 & 15786.985 & 27249.544 & -1.525 & 39 \\
\hline 8964.047 & 12219.976 & 23372.581 & -2.367 & 54 \\
\hline 9107.654 & 18118.701 & 29095.464 & -1.871 & 51 \\
\hline 9109.794 & 9711.962 & 20686.146 & -2.989 & 14 \\
\hline 9233.273 & 10855.323 & 21682.747 & -2.525 & 56 \\
\hline 9267.086 & 8460.352 & 19248.270 & -2.237 & 48 \\
\hline 9393.774 & 8605.841 & 19248.270 & -3.158 & 51 \\
\hline 9876.691 & 13250.508 & 23372.581 & -2.044 & 54 \\
\hline 10045.999 & 15236.637 & 25188.120 & -2.521 & 51 \\
\hline 10169.301 & 10855.323 & 20686.146 & -2.714 & 14 \\
\hline
\end{tabular}

Table 3. continued.

\begin{tabular}{rrrrr}
\hline \hline $\begin{array}{r}\lambda_{\text {air }} \\
(\AA)\end{array}$ & $\begin{array}{r}\text { Lower level } \\
\left(\mathrm{cm}^{-1}\right)\end{array}$ & $\begin{array}{r}\text { Upper level } \\
\left(\mathrm{cm}^{-1}\right)\end{array}$ & $\log g f$ & Unc. $^{a}$ \\
\hline 10219.381 & 14349.388 & 24132.035 & -1.982 & 50 \\
10356.371 & 15786.985 & 25440.231 & -2.163 & 51 \\
10360.000 & 9400.964 & 19050.829 & -2.143 & 49 \\
10404.508 & 8605.841 & 18214.426 & -2.311 & 51 \\
10483.366 & 9711.962 & 19248.270 & -2.356 & 51 \\
10834.612 & 15236.637 & 24463.789 & -2.200 & 51 \\
11521.826 & 15786.985 & 24463.789 & -2.147 & 51 \\
11911.506 & 10855.323 & 19248.270 & -2.287 & 51 \\
12684.806 & 13250.508 & 21131.799 & -2.433 & 55 \\
\hline
\end{tabular}

${ }^{a}$ Uncertainty in $\%$ in the $f$-value.

significant lines were predicted to fall outside the wavelength interval covered in the measurements. The residuals are therefore assumed to consist of lines too weak to be measured in the spectra. Since the branching ratio of the weakest lines measured is less than $1 \%$, the residual $\mathrm{BF}$ contains lines weaker than this. The total residual $\mathrm{BF}$ is estimated to be small for levels with short lifetime. Table 3 is a finding list including all $\log g f$-values reported in this paper sorted by wavelength.

\section{Conclusion}

In order to improve the quality of the atomic data used in cosmochronometers we have measured absolute oscillator strengths for 180 Th II lines by combining experimental values of branching fractions and radiative lifetimes.

The $f$-values obtained by this method can be used for a detailed abundance analysis of thorium in high-resolution spectra of stars enriched in heavy elements. The data will be applied to the metal poor star CS 31082-001, for which an age has been determined by using U II and Th II lines (Cayrel et al. 2001). The presence of thorium in this star is illustrated in Fig. 2. The stellar spectrum was obtained at the European Southern Observatory in Chile with the VLT telescope and UVES spectrograph. The resolving power is 70000 and the signal to noise ratio is 150 at $3859 \AA$ and 250 at $6000 \AA$.

With the present Th II data and simultaneously measured data for U II (Nilsson et al. 2001) it is now possible to make an improved determination of the age and decrease the uncertainty.

Acknowledgements. This work was supported by the Crafoord Foundation (HL), the Swedish National Space Board (SJ) and the Swedish Natural Science Research Council (SJ). We gratefully acknowledge the support from Prof. S. Svanberg and Lund Laser Centre. The authors would also like to thank Dr. V. Hill 


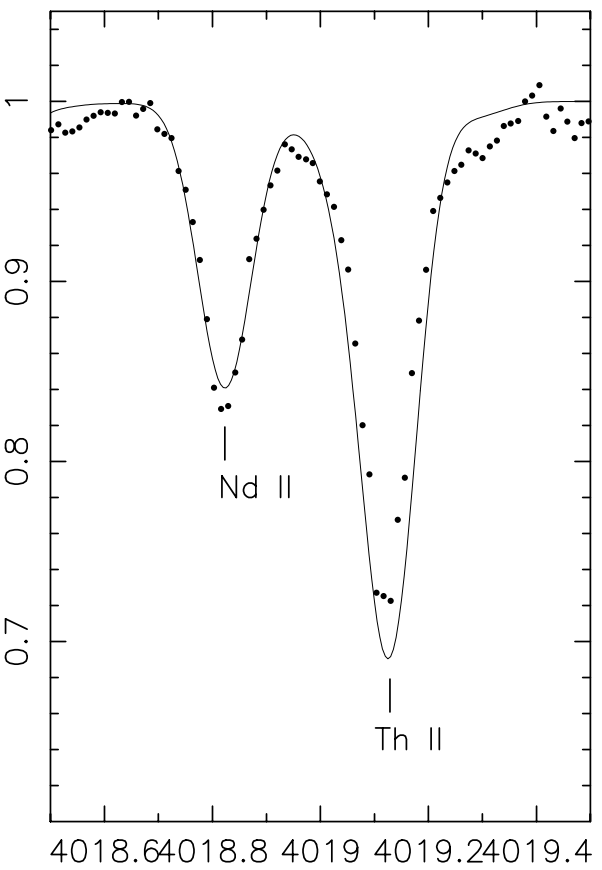

$\lambda(\AA)$

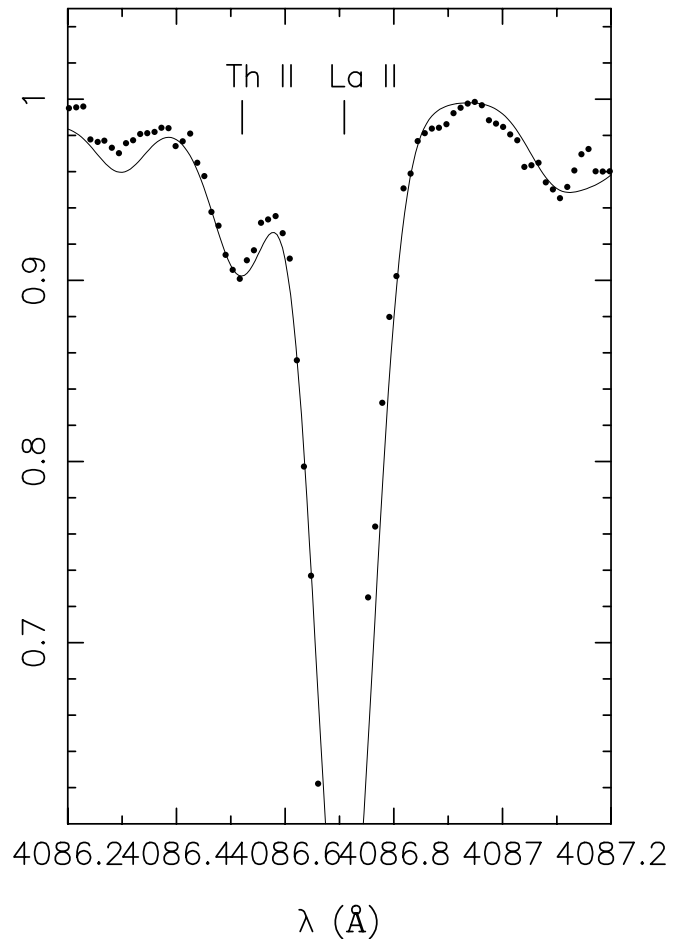

$\lambda(\AA)$

Fig. 2. Small spectral intervals including two of the strong Th II lines in the spectrum of CS 31082-001. The dotted line shows the recorded data points while the solid line shows a synthetic spectrum.

for preparing Fig. 2 and Prof. U. Litzén for his advice and assistance concerning the branching fraction measurements.

\section{References}

Andersen, T., \& Petkov, A. P. 1975, A\&A, 45, 237

Blaise, J., \& Wyart, J. F. 1992, Energy levels and atomic spectra of actinides, International tables of selected constants, Publication subsidised by: The Frensh Centre National de la Recherche Scientifique and Belgian Government Cayrel, R., Hill, V., Beers, T. C., et al. 2001, Nature, 409, 691 Corliss, C. H. 1979, MNRAS, 189, 607

Corliss, C. H., \& Bozman, W. R. 1962, NBS monograph, vol. 53 (Washington, DC: US Govt Pritning Office)
Cowan, R. D. 1981, The Theory of Atomic Structure and Spectra (University of California Press, Berkeley 1981), The Cowan code is available at different Web sites, e.g. http://plasma-gate.weismann.ac.il, where a version modified for PC by Ralchenko and Kramida can be found

Li, Z. S., Lundberg, H., Wahlgren, G. M., \& Sikström, C. M. 2000, Phys. Rev., A62, 032505

Nilsson, H., Ivarsson, S., Johansson, S., \& Lundberg, H. 2001, A\&A, submitted

Palmer, B. A., \& Engleman, Jr. R. 1983, LA-9615, Los Alamos Internal Report

Sikström, C. M., Nilsson, H., Litzèn, U., Blom, A., \& Lundberg, H. 2001, accepted by J. Quant. Spectrosc. Radiat. Transfer

Simonsen, H., Worm, T., Jessen, P., \& Poulsen, O. 1988, Phys. Scr., 38,370

Whaling, W., Carle, M. T., \& Pitt, M. L. 1993, J. Quant. Spectrosc. Radiat. Transfer, 50, 7 